\title{
Detecting horizontal gradient of sound speed in ocean
}

\author{
Motoyuki Kido \\ RCPEV, Graduate School of Science, Tohoku Univ., Aoba-ku, Sendai, 980-8578, Japan \\ (Received June 15, 2007; Revised July 23, 2007; Accepted July 25, 2007; Online published August 30, 2007)
}

\begin{abstract}
We propose a new approach to monitor the horizontal gradient of sound speed in ocean for its correction on seafloor positioning using the GPS/acoustic technique. The new method requires five seafloor transponders to solve five parameters: $\delta \boldsymbol{x}$, horizontal position of a transponder array; $\delta t$, a common delay in traveltimes due to the stratified component of sound speed; $\nabla_{t}$, the gradient of traveltime delays among the transponders associated with the sound speed gradient. We also numerically evaluate the geometrical strength of the five transponders' layout and observation point to avoid possible trade-off among the parameters.
\end{abstract}

Key words: GPS/Acoustic technique, seafloor geodesy, sound speed, inverse problem.

\section{Introduction}

Since the seafloor positioning technique was presented (Spiess, 1985) and demonstrated (Spiess et al., 1998), several research groups have developed observation systems at regions attracting their interest. Most of the up-to-date scientific products related to the monitoring of crustal deformation near plate boundaries and coseismic displacements are reported by Gagnon et al. (2005), Fujita et al. (2006), Kido et al. (2006a), Tadokoro et al. (2006), Matsumoto et al. (2006), and Chadwell and Spiess (2007), among others.

The details of the system differ among the research groups; however, the essence is in common: seafloor position is indirectly observed through acoustic ranging between three or more seafloor transponders and a surface transducer, which is usually equipped on a research vessel or a floating buoy and whose position is monitored by the kinematic GPS technique. In the present analytic scheme, sound speed in the ocean is assumed to be laterally stratified and its vertically averaged quantity is simultaneously solved with the position of the seafloor transponder array (Fujita $e t$ al., 2006; Kido et al., 2006b; Sugimoto et al., 2006). With this scheme, violation of the sound speed stratification immediately reflects the apparent position of the transponders or their array.

Several causes of the violation are expected, depending on their time-scales. Short time-scale but steadily periodical undulation may be generated by an internal gravitational wave, which is the oscillation of stratified density interface(s) within the ocean excited by tidal flow over topographic high or surface wind forcing. Propagation of this internal wave can be revealed in detail by recent technologies, such as remote-sensing from the space, and even using acoustic reflection signals with a seismic exploration technique (Holbrook and Fer, 2005). In contrast, long and un-

Copy right (C) The Society of Geomagnetism and Earth, Planetary and Space Sciences (SGEPSS); The Seismological Society of Japan; The Volcanological Society of Japan; The Geodetic Society of Japan; The Japanese Society for Planetary Sciences; TERRAPUB steady temporal variation may be due to the advection of anomalous seawater driven by tidal flow or oceanic current and, sometimes, the change in the current axis itself. The former undulation does not affect the apparent array position for its short time-scale after taking the temporal average, while the latter has a possibility to make a significant error in the positioning.

\section{Describing Sound Speed Structure}

Inferring sound speed, the description of its horizontal gradient is not straightforward. First, we define $\delta t$, the total delay of traveltime for the vertical path due to change in laterally stratified sound speed, as

$$
\delta t=\int_{0}^{Z} \delta s(z) \mathrm{d} z=\sum_{n=1}^{N} \delta s_{n}\left(z_{n}-z_{n-1}\right),
$$

where $\delta s(z)$ is the deviation of slowness from a reference depth profile from surface $(z=0)$ to the bottom $(z=Z)$. The right-hand term is its discretized expression from the surface $\left(z_{0}=0\right)$ to the bottom $\left(z_{N}=Z\right) . \delta s(z)$ at $z>$ $1000 \mathrm{~m}$ is negligible in most cases. We assume that any horizontal variation in sound speed has a wavelength that is long enough to be approximated by linear functions. Here we consider two extreme cases, as shown in Fig. 1(a, b). In Fig. 1(a), slowness $s_{n}$ in each layer does not change in the horizontal and, alternatively, the layer depth $z_{n}$ can linearly change with $x$. In this case, total delay in traveltime (normalized to vertical component through $\cos \xi_{k}$ ) between surface transducer at $x_{0}$ and $k$-th seafloor transponder at $x_{k}$ purely due to the set of inclined layers is expressed as

$$
\frac{x_{k}-x_{0}}{z_{N}} \sum_{n=1}^{N-1}\left(s_{n}-s_{n+1}\right) z_{n} \frac{\partial z_{n}}{\partial x}=\nabla_{t} \cdot\left(x_{k}-x_{0}\right) .
$$

In Fig. 1(b), $s_{n}$ itself changes with $x$ while $z_{n}$ remains unchanged. Delay in the $n$-th layer is $\int_{z_{n-1}}^{z_{n}} \frac{\partial s_{n}}{\partial x} \frac{x_{k}-x_{0}}{z_{N}} z \mathrm{~d} z$ and then the total delay can be written as

$$
\frac{x_{k}-x_{0}}{2 z_{N}} \sum_{n=1}^{N}\left(z_{n}^{2}-z_{n-1}^{2}\right) \frac{\partial s_{n}}{\partial x}=\nabla_{t} \cdot\left(x_{k}-x_{0}\right) .
$$


(a)

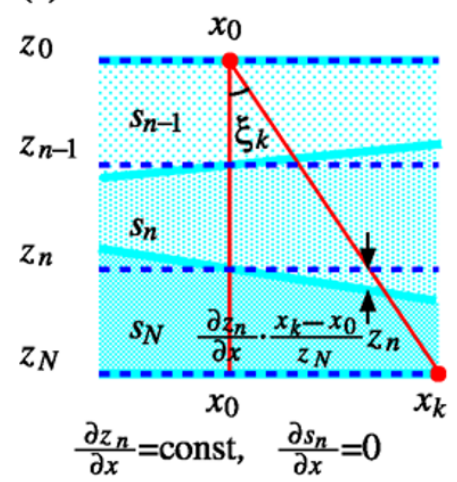

(b)

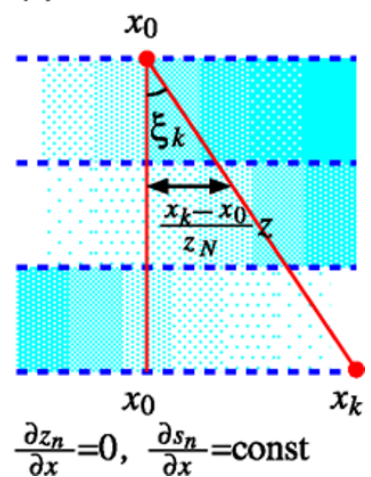

Fig. 1. Horizontal gradient of sound speed structure for two extreme cases: (a) only the depth of each layer $z_{n}$ changes and (b) only the slowness $s_{n}$ changes with $x$.

In both of these extreme cases, the delay is expressed by the linear function of the horizontal distance $x_{k}-x_{0}$ with a constant denoted by $\nabla_{t}$, which is the effective contribution of the sound speed gradient on traveltime. This justifies the use of the linear expression of the gradient in the observation equation in the next section.

\section{Observation Equation}

As described in Spiess (1985), only a single but simultaneous acoustic ranging to three transponders can determine relative displacement of a transponder array in the conditions that: sound speed is laterally stratified; the transponder array is rigid and can move only horizontally. In other words, array displacement can be monitored as a timeseries, even with temporally varying sound speed.

In the general two-dimensional (horizontal) case, the observation equation at a certain time is defined as follow:

$$
\begin{aligned}
& {\left[t_{k}^{\mathrm{obs}}-t_{k}^{\mathrm{cal}}\left(\boldsymbol{x}_{0}, \boldsymbol{x}_{k}+\delta \boldsymbol{x}, s_{0}(z)\right)\right] \cos \xi_{k}} \\
& \quad-\delta t-\boldsymbol{\nabla}_{t} \cdot\left(\boldsymbol{x}_{k}-\boldsymbol{x}_{0}\right)=0 \quad(k=1,2, \ldots, K)
\end{aligned}
$$

where $t_{k}^{\mathrm{obs}}$ and $t_{k}^{\mathrm{cal}}$ respectively are observed and synthetic traveltimes to $k$-th transponder. $\boldsymbol{x}_{k}$ is the initial position of $k$-th transponder accompanied by displacement vector $\delta \boldsymbol{x}$, which is common for all the transponders as well as $\delta t$ and $\nabla_{t} . s_{0}(z)$ is the depth profile of reference slowness for synthetic calculation. The equation is identical to Eq. (1) in Kido et al. (2006a), with the exception for the additional term of horizontal gradient. In Eq. (4), the number of unknowns is five: $\delta \boldsymbol{x}=(\delta x, \delta y), \delta t$, and $\nabla_{t}=\left(\nabla_{t_{x}}, \nabla_{t_{y}}\right)$. As such, at least five transponders $(K=5)$ are required to solve the five parameters, while only three transponders are necessary for the traditional assumption of laterally stratified sound speed.

\section{One-dimensional Case}

In the evaluation of response of vertically normalized traveltime $t_{k}$ by $\cos \xi_{k}$ against $\delta \boldsymbol{x}, \delta t$, and $\boldsymbol{\nabla}_{t}$, we first consider a simple one-dimensional case, which, for example, accounts only for east-west component using linearly aligned three transponders $(K=3)$. The partial derivative of $t_{k}$ with respect to $\delta x$ can be obtained by simple geometric

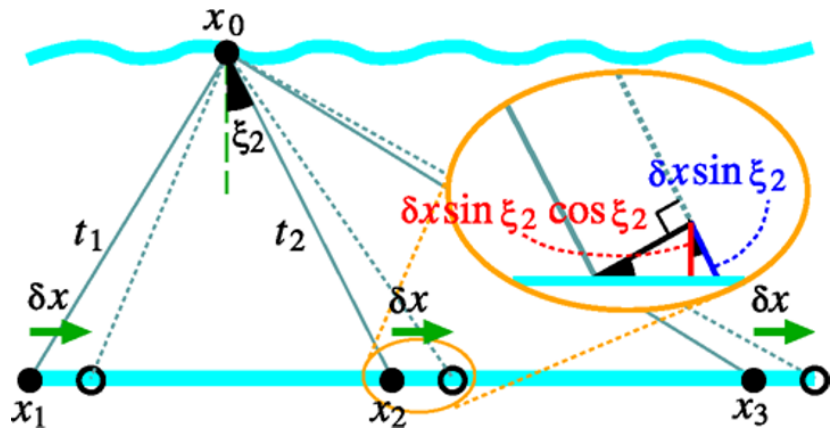

Fig. 2. Illustration of change in the vertical component of slant range due to horizontal shift of the linearly aligned three seafloor transponders by $\delta x$.

construction (Fig. 2), as well with respect to $\delta t$ and $\nabla_{t}$ by their definition in Eq. (4):

$$
\frac{\partial t_{k}}{\partial \delta x}=s_{0} \sin \xi_{k} \cos \xi_{k}, \quad \frac{\partial t_{k}}{\partial \delta t}=1, \quad \frac{\partial t_{k}}{\partial \nabla_{t}}=x_{k}-x_{0}
$$

Here we define a response matrix $\boldsymbol{R}$, whose elements are partial derivatives of $t_{k}$ and are normalized by vertically averaged reference slowness $\bar{s}_{0}$ or seafloor depth $Z$ for nondimentionalization:

$$
\boldsymbol{R}=\left(\begin{array}{lll}
\boldsymbol{r}_{\delta x} & \boldsymbol{r}_{\delta t} & \boldsymbol{r}_{\nabla_{t}}
\end{array}\right)
$$

where

$$
\begin{aligned}
\boldsymbol{r}_{\delta x} & =\left(\frac{\partial t_{1}}{\partial \delta x}, \frac{\partial t_{2}}{\partial \delta x}, \frac{\partial t_{3}}{\partial \delta x}\right)^{T} \cdot \frac{1}{\bar{s}_{0}} \\
\boldsymbol{r}_{\delta t} & =\left(\frac{\partial t_{1}}{\partial \delta t}, \frac{\partial t_{2}}{\partial \delta t}, \frac{\partial t_{3}}{\partial \delta t}\right)^{T} \\
\boldsymbol{r}_{\nabla_{t}} & =\left(\frac{\partial t_{1}}{\partial \nabla_{t}}, \frac{\partial t_{2}}{\partial \nabla_{t}}, \frac{\partial t_{3}}{\partial \nabla_{t}}\right)^{T} \cdot \frac{1}{Z}
\end{aligned}
$$

$\boldsymbol{R}$ is a $3 \times 3$ square matrix to solve linearized observation equation with $\Delta t_{k}$, the changes in $t_{k}$ :

$$
\boldsymbol{R}\left(s_{0} \delta x, \delta t, Z \nabla_{t}\right)^{T}=\left(\Delta t_{1}, \Delta t_{2}, \Delta t_{3}\right)^{T}
$$

$\boldsymbol{R}$ depends on the observation point $x_{0}$. When response vectors, $\boldsymbol{r}_{\delta x}, \boldsymbol{r}_{\delta t}$, and $\boldsymbol{r}_{\nabla_{t}}$, are not linearly independent, the equation is no longer able be solved. Trade-off between individual pair of parameters can be evaluated using the length of their cross product because it becomes zero when two vectors are in the same direction. It is clear that $\left|\boldsymbol{r}_{\nabla_{t}} \times \boldsymbol{r}_{\delta x}\right|$ indicated by the thin blue line in Fig. 3 is zero at the center of the array $\left(x_{0}=x_{2}\right)$. This means that one can not distinguish $\delta x$ from $\nabla_{t}$ at this observation position, while $\delta t$ is well resolved. The degree of total geometrical strength can be diagnosed by way of a well-known quantity $\kappa(\boldsymbol{R})$, the condition number of the matrix $\boldsymbol{R}$ (e.g., Golub and Van Loan, 1996):

$$
\kappa(\boldsymbol{R})=\|\boldsymbol{R}\|_{2}\left\|\boldsymbol{R}^{-1}\right\|_{2}=\frac{\sigma_{\max }(\boldsymbol{R})}{\sigma_{\min }(\boldsymbol{R})},
$$

where $\|\boldsymbol{R}\|_{2}$ is the $L_{2}$-norm of the matrix $\boldsymbol{R}$, and $\sigma_{\max }(\boldsymbol{R})$ and $\sigma_{\min }(\boldsymbol{R})$ the maximum and minimum singular values of $\boldsymbol{R}$, obtained through singular value decomposition. This 


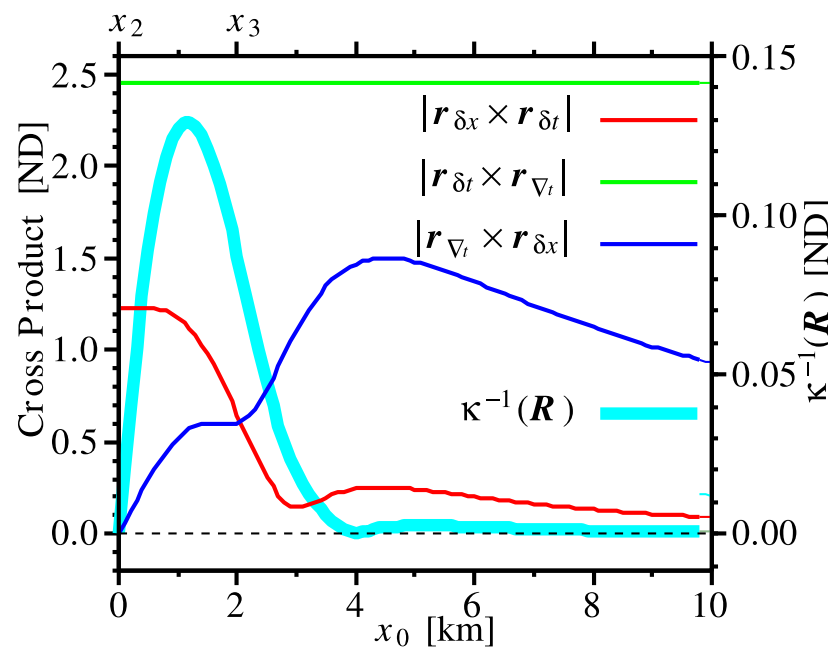

Fig. 3. Plot of the reciprocal condition number $\kappa^{-1}(\boldsymbol{R})$ for evaluation of the geometrical strength (thick line) in the case of seafloor depth $Z=2 \mathrm{~km}$ and three transponders equidistantly aligned at $x=-2,0,2 \mathrm{~km}$, respectively. $L_{2}$-norms of cross product between response vectors (thin lines) are also shown to diagnose trade-off between individual pair of the parameters. Only $x_{0} \geq 0$ is plotted for it symmetry.

quantity represents the degree of the propagation of traveltime error into estimate of the parameters. Reciprocal condition number $\kappa^{-1}(\boldsymbol{R})$ is 1 for the idealized problem and zero for the extremely ill-condition. $\kappa^{-1}(\boldsymbol{R})$ is plotted with a thick line for varying $x_{0}$ in Fig. 3 . The best condition is observed at a position departs from the center of the array $\left(x_{0}=x_{2}\right)$, which is in accordance with the behavior of the three cross products.

\section{General Two-dimensional Case}

The arguments introduced in the previous section can be easily extended to a two-dimensional case. The displacement of transponders and the gradient of sound speed split into vectors of two horizontal components, as in Eq. (4). The total number of parameters increases to five, which requires five transponders $(K=5)$ as well. The number of cross product pairs is ${ }_{5} C_{2}=10$ and it is not practical to diagnose all the combinations. Therefore, we evaluate only the condition number in the two-dimensional case. The response matrix $\boldsymbol{R}$ given in Eq. (6) is extended to a $5 \times 5$-square matrix, which consists of $\boldsymbol{r}_{\delta x}, \boldsymbol{r}_{\delta y}, \boldsymbol{r}_{\delta t}, \boldsymbol{r}_{\nabla_{t x}}$, and $\boldsymbol{r}_{\nabla_{t y}}$ for five transponders $(k=1, \ldots, 5), \boldsymbol{r}_{\delta x}$ and $\boldsymbol{r}_{\delta y}$ are easily formulated with a geometrical construction in the analogy with the onedimensional case and the other vectors come from Eq. (4).

There is a variety of choice to arrange five transponders on the two-dimensional seafloor. We employ here four examples to evaluate geometrical strength $\kappa^{-1}(\boldsymbol{R})$ for varying observation point $\boldsymbol{x}_{0}$, as shown in Figs. $4(\mathrm{a}-\mathrm{d})$. Better conditions are expected at $\boldsymbol{x}_{0}$ with larger $\kappa^{-1}(\boldsymbol{R})$. The largest value of 0.095 is observed in the L-shaped layout, which is not so small compared to the largest value of 0.12 in the one-dimensional case. The areas of poor geometrical strength lie distant from the array and along the regions of trade-off between $\delta \boldsymbol{x}$ and $\boldsymbol{\nabla}_{t}$, which is similar to the onedimensional case. To distinguish these two parameters, it is important to observe traveltimes at a region having different incident angles and directions to transponders.

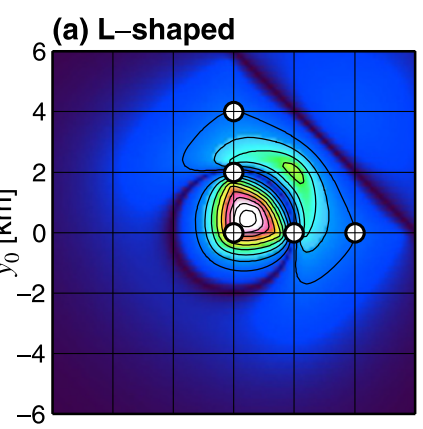

(c) Pentagon

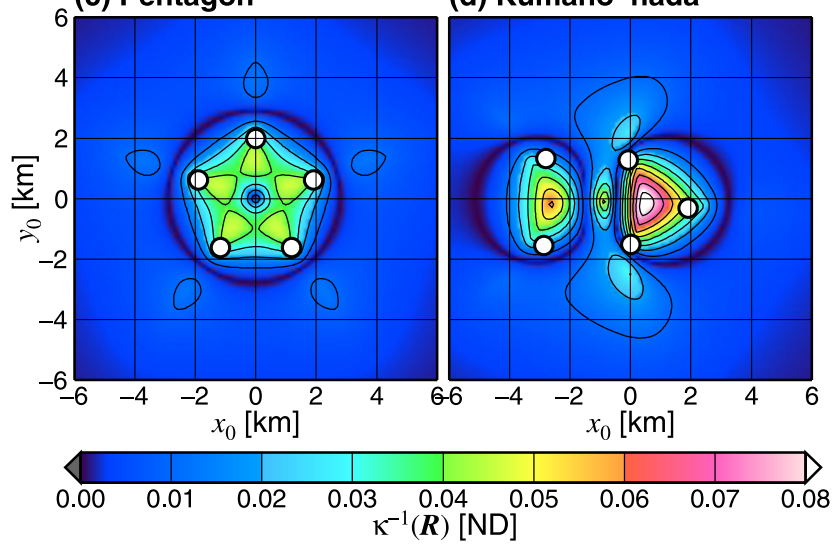

Fig. 4. Plot of $\kappa^{-1}(\boldsymbol{R})$ as a geometrical strength for varying observation point at $\boldsymbol{x}_{0}=\left(x_{0}, y_{0}\right)$. Four sets of layout using five transponders (open circles) are examined. Seafloor depths are set to $2 \mathrm{~km}$ with an exception for (d) Kumano-nada, with an actual depth of $2030 \mathrm{~m}$ at this site. Smaller $\kappa^{-1}(\boldsymbol{R})$ (blue region) indicates position of ill-condition.

\section{Discussion}

We have proposed an idea to simultaneously solve the position of transponder array $\delta \boldsymbol{x}$, the common and individual component of traveltime delays, $\delta t$ and $\nabla_{t}$, which respectively reflect the stratified component and gradient of the integrated sound speeds. Using this method, we can distinguish $\nabla_{t}$ from $\delta \boldsymbol{x}$, while both effects are projected onto only $\delta \boldsymbol{x}$ in the past analytic scheme.

Introducing six or more transponders will give redundancy in the problem and hence results in higher geometrical strength. On the one hand, it is impractical to employ higher-order representation of horizontal sound speed variation with these extra transponders since the problem is then ill-posed with too many parameters. This is the difference with the ocean acoustic tomography (Munk et al., 1995), which can efficiently exploit multipath information due to reflections at the surface and refraction in the SOFAR channel. The linear representation in this study may not be adequate for small-scale structure in sound speed, such as warm or cold eddies, that irregularly appear in the sea surface. However, these local anomalies hardly keep their position and may pass over the survey site in a relatively short time.

In contrast to the local anomalies, long-lived and longwavelength anomalies contribute a serious error in apparent position of the transponder array, when the horizontal gradient of sound speed is not taken into account. In the traditional survey, we usually continue measurements for more than 1 day or sometimes up to a couple of days in order 
to take temporal average of the apparent position for long enough period to eliminate the above effect. The linear approximation employed in this study is adequate to represent such a horizontally large-scale feature. If we distinguish the effect from the apparent position using the new analytic scheme, we can significantly reduce the survey time. This is crucial, especially for semi-realtime monitoring of the crustal displacement using an on-line seafloor cable of the DONET project in the future (Kawaguchi et al., 2007).

There are two kinds of error which deteriorate the analysis. One is transducer position associated with kinematic GPS error or attitude monitoring of the buoy, and the other is traveltime reading due to noise in acoustic signals. The former causes systematic error depending on its origin. It should be noted that the former error separately affects the parameter estimation. Theoretically, with exact traveltimes, error in the horizontal component of transducer positioning is directly and only projected onto $\delta \boldsymbol{x}$ while the vertical component only affects $\delta t$. This behavior is due to the vertical normalization using incident elevation angles in describing any parameters. In contrast, the latter simultaneously affects the estimate in all the parameters.

Here we consider the detectable level of the parameters in terms of propagation of traveltime errors. When Eq. (10) is symbolically denoted as $\boldsymbol{R} \boldsymbol{m}=\boldsymbol{d}$, the propagation of errors in traveltimes $\delta \boldsymbol{d}$ to the parameters $\delta \boldsymbol{m}$ can be written as

$$
\begin{aligned}
\boldsymbol{R}(\boldsymbol{m}+\delta \boldsymbol{m}) & =\boldsymbol{d}+\delta \boldsymbol{d} \\
\delta \boldsymbol{m} & =\boldsymbol{R}^{-1} \delta \boldsymbol{d}
\end{aligned}
$$

Using a pulse-compressed acoustic signal of $10 \mathrm{kHz}$ in frequency and the correlating technique of transmitted and received signals, which are widely used in this field, we achieve an accuracy in traveltime detection better than $10 \mu \mathrm{s}$ and hence $5 \mu \mathrm{s}$ for a one-way traveltime as far as the correct peak in correlogram being identified. Applying normally distributed errors of $5 \mu$ s standard deviation to $\delta \boldsymbol{d}$, we statistically obtain standard deviations of the individual parameters in $\delta \boldsymbol{m}$ from Eq. (13) that are 2.74 and $2.57 \mathrm{~cm}$ in $\delta x$ and $\delta y, 2.92 \mu \mathrm{s}$ in $\delta t$, and 3.18 and $4.15 \mu \mathrm{s} / \mathrm{km}$ in $\nabla_{t_{x}}$ and $\nabla_{t_{y}}$ for the case of the maximum $\kappa^{-1}(\boldsymbol{R})=0.087$ in Fig. 4(d) at $\left(x_{0}, y_{0}\right)=(400,-200) \mathrm{m}$. The randomicity of $\delta \boldsymbol{d}$ elements will statistically cancel their effect, which results in the smaller propagation error in $\delta t$ than the given errors in $\delta \boldsymbol{d}$. For example, $4.15 \mu \mathrm{s} / \mathrm{km}$ in $\nabla_{t_{y}}$ corresponds to roughly $2.5 \mathrm{~cm}$ of mis-positioning in $\delta y$ through Eq. (5) when $\nabla_{t_{y}}$ is projected onto $\delta y$ in the traditional analytic scheme. The fluctuation of the apparent position in our past survey (Kido et al., 2006a) indicates a much larger horizontal gradient. In addition, because of the random nature in the traveltime error, its effect on the parameter estimation decreases after taking a time average for a certain period. Therefore, we consider that detecting $\boldsymbol{\nabla}_{t}$ is possible when measurements are made at a proper position suggested in Fig. 4. We emphasize again that the positioning error in the transducer does not affect the $\nabla_{t}$ while it is superimposed on $\delta \boldsymbol{x}$ and $\delta t$.

We also applied the analyses to the traditional survey design of three transponders of an equilateral triangle array for stratified sound speed. The best observation position is found at the center of the array, where $\kappa^{-1}(\boldsymbol{R})=0.35$. Although $\kappa^{-1}(\boldsymbol{R})$ is roughly four times larger than that for the five transponders shown above, the error propagations are improved only by half for $\delta \boldsymbol{x}$ and are nearly unchanged for $\delta t$. Our past analysis in the traditional scheme well resolved $\delta t$ in comparison with in situ sound speed measurements (Kido et al., 2006b). This also encourages the feasibility of this research.

In practice, our research group has five transponders at Kumano-nada, Japan (Kido et al., 2006a) as indicated in Fig. 4(d). This site was intended to form two arrays, a square in the west and a triangle in the east, in the traditional analytic scheme of stratified sound speed. For this reason, we have not made traveltime measurement to the five transponders all together. We plan to carry out simultaneous ranging to the five transponders in the next survey to confirm the idea proposed in this paper.

Acknowledgments. The author thanks Mr. Hiroaki Tsushima for discussion on the geometrical strength of the problem. Review comments by Dr. Yoshihiro Matsumoto and an anonymous reviewer significantly improve the manuscript. This research is supported by the DONET program, MEXT, Japan.

\section{References}

Chadwell, C. D. and F. N. Spiess, Plate motion at the ridge-transform boundary of the south Cleft segment of the Juan de Fuca Ridge from GPS-Acoustic data, J. Geophys. Res., 2007 (submitted).

Fujita, M., T. Ishikawa, M. Mochizuki, M. Sato, S. Toyama, M. Katayama, K. Kawai, Y. Matsumoto, T. Yabuki, A. Asada, and O. L. Colombo, GPS/Acoustic seafloor geodetic observation: method of data analysis and its application, Earth Planets Space, 58, 265-275, 2006.

Gagnon, K., C. D. Chadwell, and E. Norabuena, Measuring the onset of locking in the Peru-Chile trench with GPS and acoustic measurements, Nature, 434, 205-208, 2005.

Golub, G. H. and C. F. Van Loan, in Matrix Computations (3rd ed.), Johns Hopkins University Press, Baltimore, MD, 694 pp., 1996.

Holbrook, W. S. and I. Fer, Ocean internal wave spectra inferred from seismic reflection transects, Geophys. Res. Lett., 32, doi:10.1029/2005GL 023733, 2005.

Kawaguchi, K., E. Araki, and Y. Kaneda, A design concept of seafloor observatory network for earthquakes and tsunamis, abstract in International Symposium on Underwater Technology 2007, Tokyo, 176-178, 2007.

Kido, M., H. Fujimoto, S. Miura, Y. Osada, K. Tsuka, and T. Tabei, Seafloor displacement at Kumano-nada caused by the 2004 off Kii Peninsula earthquakes, detected through repeated GPS/Acoustic surveys, Earth Planets Space, 58, 911-915, 2006a.

Kido, M., Y. Osada, and H. Fujimoto, On the relation of radially-averaged sound velocities in seawater, measured with XBT and estimated in acoustic ranging, abstract for 106th Meeting of the Geodetic Society of Japan, 2006b (in Japanese).

Matsumoto, Y., M. Fujita, T. Ishikawa, M. Mochizuki, T. Yabuki, and A. Asada, Undersea co-seismic crustal movements associated with the 2005 Off Miyagi Prefecture Earthquake detected by GPS/acoustic seafloor geodetic observation, Earth Planets Space, 58, 1573-1576, 2006.

Munk, W., P. Worcester, and C. Wunsch, Ocean acoustic tomography, 447 pp., Cambridge Univ. Press, 1995.

Spiess, F. N., Analysis of a possible sea floor strain measurement system, Marine Geodesy, 9, 385-398, 1985.

Spiess, F. N., C. D. Chadwell, J. A. Hildebrand, L. E. Young, G. H. Purcell Jr., and H. Dragert, Precise GPS/Acoustic positioning of seafloor reference points for tectonic studies, Phys. Earth Planet. Inter., 108, 101-112, 1998.

Sugimoto, S., R. Ikuta, M. Ando, K. Tadokoro, T. Okuda, and G. M. Besana, Evaluation for GPS/Acoustic seafloor positioning based on repeated CTD measurements, Earth Planets Space, 2006 (submitted).

Tadokoro, K., M. Ando, R. Ikuta, T. Okuda, G. Besana, S. Sugimoto, and M. Kuno, Observation of coseismic seafloor crustal deformation due to M7 class offshore earthquakes, Geophys. Res. Lett., 33, doi:10.1029/2006GL026742, 2006.

M. Kido (e-mail: kido@aob.geophys.tohoku.ac.jp) 